\title{
Engineered Tumor-Derived Extracellular Vesicles: Potentials in Cancer Immunotherapy
}

\author{
Adeleh Taghikhani1,2, Farzin Farzaneh ${ }^{3}$, Farzaneh Sharifzad ${ }^{2,4}$, Soura Mardpour ${ }^{2,5}$, \\ Marzieh Ebrahimi2* and Zuhair Mohammad Hassan ${ }^{1 *}$
}

\begin{abstract}
${ }^{1}$ Department of Immunology, Faculty of Medical Sciences, Tarbiat Modares University, Tehran, Iran, ${ }^{2}$ Department of Stem Cells and Developmental Biology, Cell Science Research Center, Royan Institute for Stem Cell Biology and Technology, ACECR, Tehran, Iran, ${ }^{3}$ Division of Cancer Studies, Department of Haematological Medicine, King's College London, London, United Kingdom, ${ }^{4}$ Department of Applied Cell Sciences, Kashan University of Medical Sciences, Kashan, Iran, ${ }^{5}$ Department of Tissue Engineering and Applied Cell Sciences, School of Advanced Technologies in Medicine, Tehran University of Medical Sciences, Tehran, Iran
\end{abstract}

\section{OPEN ACCESS}

Edited by:

Fatemeh Momen-Heravi, Columbia University, United States

Reviewed by:

Gabriele Multhoff,

Technical University of Munich,

Germany

Zong Sheng Guo,

University of Pittsburgh, United States

*Correspondence:

Marzieh Ebrahim

mebrahimi@royaninstitute.org Zuhair Mohammad Hassan

hasan_zm@modares.ac.ir

Specialty section:

This article was submitted to Cancer Immunity and Immunotherapy, a section of the journal

Frontiers in Immunology

Received: 14 May 2019 Accepted: 27 January 2020

Published: 06 March 2020

Citation:

Taghikhani A, Farzaneh F, Sharifzad F, Mardpour S, Ebrahimi M and Hassan ZM (2020) Engineered Tumor-Derived Extracellular Vesicles: Potentials in Cancer Immunotherapy.

Front. Immunol. 11:221. doi: 10.3389/fimmu.2020.00221
Exosomes are nano vesicles from the larger family named Extracellular Vesicle (EV)s which are released by various cells including tumor cells, mast cells, dendritic cells, B lymphocytes, neurons, adipocytes, endothelial cells, and epithelial cells. They are considerable messengers that can exchange proteins and genetic materials between the cells. Within the past decade, Tumor derived exosomes (TEX) have been emerged as important mediators in cancer initiation, progression and metastasis as well as host immune suppression and drug resistance. Although tumor derived exosomes consist of tumor antigens and several Heat Shock Proteins such as HSP70 and HSP90 to stimulate immune response against tumor cells, they contain inhibitory molecules like Fas ligand (Fas-L), Transforming Growth Factor Beta (TGF- $\beta$ ) and Prostaglandin E2 (PGE2) leading to decrease the cytotoxicity and establish immunosuppressive tumor microenvironment (TME). To bypass this problem and enhance immune response, some macromolecules such as miRNAs, HSPs and activatory ligands have been recognized as potent immune inducers that could be used as anti-tumor agents to construct a nano sized tumor vaccine. Here, we discussed emerging engineered exosomes as a novel therapeutic strategy and considered the associated challenges.

Keywords: cancer immunotherapy, tumor derived exosomes, engineered exosomes, immunomodulatory therapies, immunosuppression

\section{INTRODUCTION}

Although cancer immunotherapy has been stablished as a promising treatment option, it faces many challenges including the lack of recognition of specific tumor-associated antigens by the immune system, in part due to the presence of thymic tolerance to self-antigens. Moreover, the cancer microenvironment has immune suppressive properties, thus reducing responses to immune-mediated attacks against the cancer. Tumor microenvironments consist of many cell types that become educated and adapted to support primary tumor growth. Of note, tumor derived exosomes might be involved in this process. In this review, we discuss the role of Tumor derived exosomes in the associated immune suppression and the potential therapeutic applications of modified Tumor derived exosomes, which represents a novel approach for development of therapeutic cancer vaccines. 


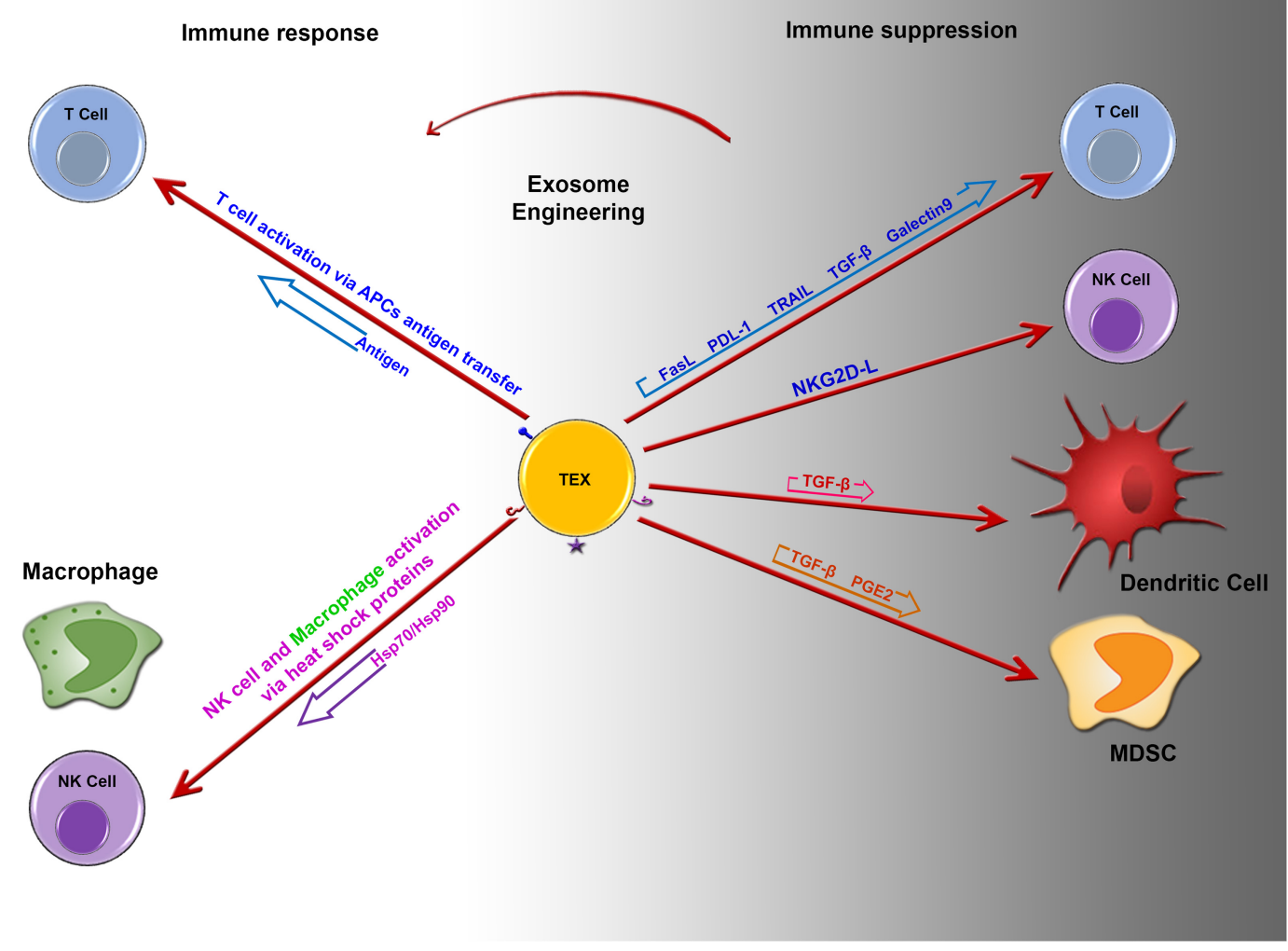

GRAPHICAL ABSTRACT | Imbalance between immune suppression and immune response by tumor derived exosomes. Potential role of engineered tumor derived exosomes in changing the fate of immune response. Image designed by Adeleh Taghikhani.

\section{TUMOR DERIVED EXOSOMES (TEXs)}

Exosomes are small vesicles $(30-120 \mathrm{~nm})$ that are derived from various cells within late endosomes. They are released into the microenvironment where they play a major role in cell-cell communication $(1,2)$. Exosomes were first considered as waste vesicular bodies of reticulocytes in process of maturation to erythrocytes (3). Moreover, recent experiments support the idea of exosomes could maintain cellular homeostasis (4).

They are generated by inward budding of multivesicular bodies (MVBs) (5), while the exact mechanism of exosome entry into the target cell is not yet clear, phagocytosis and fusion are potentially involved $(6,7)$. Exosomes are secreted by various cells. For the first time, their release was reported more than 40 years ago as platelets sprinkles (8). Immune cells and cancer cells are also recognized as exosome producers (9-12). Regarding the membrane lipid content, exosomes contain higher concentrations of sphingolipids and cholesterol than that of the cell of origin. They also contain soluble and surface proteins as well as mRNAs and miRNAs. mRNAs result in production of proteins in target cells and miRNAs are transferred between cells and affect expression of different genes (13). Proteomics analysis showed that tumor-derived exosomes (TEXs) contain major histocompatibility complex (MHC) molecules, heat shock proteins, and tetraspanin (CD63, CD81 and CD9) which are known as endosomal pathway proteins. Moreover, tumor antigens such as Mart1, gp100, TRP, and Her2-neu have been found in TEXs (9, 14); (Figure 1). Cancer cells subjected to hypoxic conditions play a role in promoting angiogenesis and metastasis by releasing potent angiogenic factors. They home to metastatic areas through the induction of molecular signals involved in tumor cell recruitment, extracellular matrix deposition, and vascular proliferation. It was also shown that some TEXs contain surface TGF- $\beta$ along with betaglycan, which could trigger SMAD-dependent signaling and regulate the differentiation of fibroblasts to myofibroblasts (15). In addition, TEXs protect cancer cells from apoptosis through the selective efflux of the apoptosis inducer proteins, which are presented by effector cells such as $\mathrm{T}$ cells or natural killer (NK) cells. Moreover, TEXs might diminish the effects of therapy via drug efflux or by masking the binding site of monoclonal antibodies; this could promote the emergence of chemotherapy-resistant cell populations (16).

\section{Biogenesis of Exosomes}

Exosome biogenesis begins with the production of endocytic vesicles via the internalization of surface lipids and clathrindependent or independent endocytosis. These vesicles can form early endosomes and late endosomes. Exosomal secretion is achieved by attaching MVBs (microvesicular bodies) to the cell 


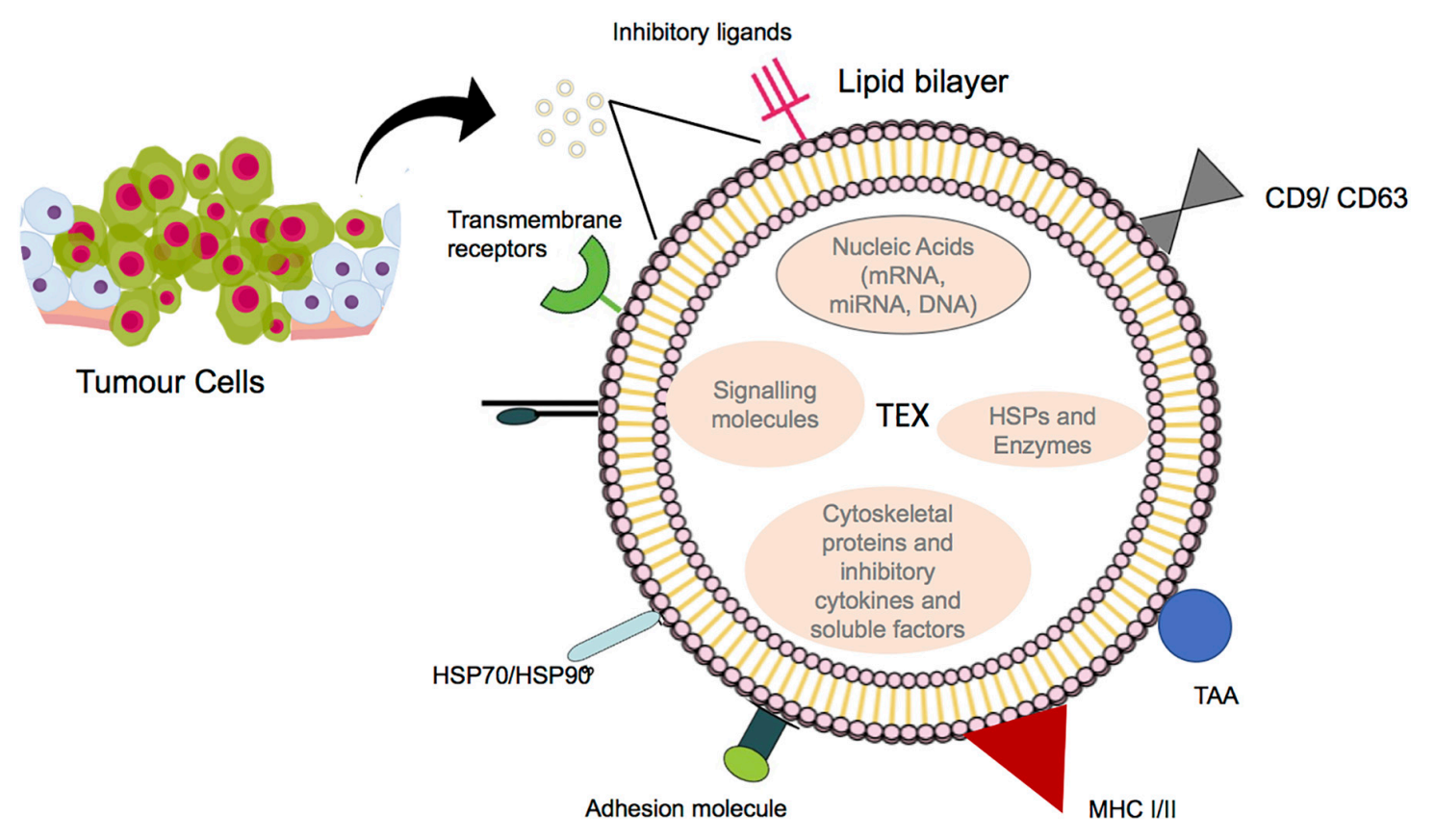

$30-120 \mathrm{~nm}$

FIGURE 1 | Structure of tumor derived exosome. Size and composition of TEXs. Image designed by Adeleh Taghikhani.

membrane, which appears to be dependent on a variety of Rab GTPase proteins and endosomal sorting complex required for transport (ESCRT). The ESCRT system assists in the sorting of endosomal ubiquitinated proteins for secretion in nanoparticles such as exosomes (17-19).

\section{Isolation and Characterization}

Differential ultracentrifugations have been considered as a gold standard method for effective isolation of extracellular vesicles from conditioned medium which is based on size. In this method, microvesicles, the vesicles which are larger than exosomes, are pelleted at $10,000-20,000 \times g$ and the supernatant is subjected to a second centrifugation step at $100,000 \times g$, which results in the isolation of exosomes that are typically smaller than microvesicles.

Other methods for exosome isolation include immunoaffinity purification (affinity chromatography), size exclusion chromatography, polymeric precipitation, microfluidics, and commercial kits based using columns or polymeric precipitations. Exosomes are characterized using a range of techniques including electron microscopy, nanoparticle tracking analysis, dynamic light scattering, flow cytometry and western blotting (20-23). Evidence has shown that the formation of EVs requires function of the endosomal sorting complex required for transport (ESCRT). The typical exosomal protein Alix, which is associated with several ESCRT (TSG101 and
CHMP4) proteins, has been reported to participate in endosomal membrane budding as well as exosomal cargo selection therefore these molecules selected as exosomal specific markers in their characterization (24).

\section{IMMUNOSUPPRESSIVE EFFECTS OF TEXs}

Tumor-derived exosomes can carry immunosuppressive molecules such as FasL, TGF- $\beta 1$, TRAIL, PD-L1, and NKG2D ligands, which are involved in suppression of the immune response (Table $\mathbf{1}$ ).

\section{Expression of FasL, PD-L1, and TRAIL by TEXs}

FasL, TRAIL (TNF-related apoptosis-inducing ligand) and PDL1 (Programmed death-ligand 1) are known as regulator of immune cells homeostasis and activity through inducing of apoptosis following receptor/ligand interactions. The tumor exosome surface may present these regulatory factors to inhibit T-cell proliferation and response through the induction of apoptosis in a dose-dependent manner. This effect can be blocked by the antibody-mediated inhibition of FasL binding to its receptor (2527). Exosomes isolated from patients with ovarian cancer could express MHC I, placental alkaline phosphatase, TRAIL, and FasL. 
TABLE 1 | Tumor-derived exosomes involved in suppression of the immune response.

\begin{tabular}{|c|c|c|}
\hline $\begin{array}{l}\text { Immunosuppressive } \\
\text { molecule }\end{array}$ & Function & References \\
\hline $\begin{array}{l}\text { Fas-L, placental } \\
\text { alkaline phosphatase, } \\
\text { B23/nucleophosmin }\end{array}$ & T cell apoptosis & $(28)$ \\
\hline Fas-L & CD8 + T cell apoptosis & $(25)$ \\
\hline Fas-L & $\begin{array}{l}\text { Induction of receptor and } \\
\text { mitochondrial apoptotic pathways } \\
\text { in Jurkat and activated T cells. }\end{array}$ & $(26)$ \\
\hline TGF- $\beta$ & Differentiation to myofibroblast & $(15)$ \\
\hline TGF- $\beta$ & T cell suppression & (29) \\
\hline NKG2D-L & NK cell suppression & $(32)$ \\
\hline NKG2D-L & NK cell down regulation & (33) \\
\hline BAG6, BAG4 & Tumor evasion & (35) \\
\hline ND & Treg induction & (39) \\
\hline ND & Treg induction & $(40)$ \\
\hline PGE2 and TGF- $\beta$ & $\begin{array}{l}\text { Induction of MDSC expressing } \\
\text { Cox2, IL-6, VEGF and arginase-1 }\end{array}$ & $(78)$ \\
\hline STAT3 & MDSC induction & $(41)$ \\
\hline IL-6 & Blockade of DC differentiation & $(43)$ \\
\hline RIG1 and STAT1 & Therapy resistancy & $(44)$ \\
\hline PGE2 & DC dysfunction & $(42)$ \\
\hline $\begin{array}{l}\text { Upregulation of } \\
\text { inhibitory genes }\end{array}$ & Functional decline in T cells & $(37)$ \\
\hline TGF- $\beta$ and PD-L1 & Drug resistance & (30) \\
\hline
\end{tabular}

These exosomes also could suppress the activatory signals of $\mathrm{T}$ cells (CD3z and JAK3) following the incubation of TEXs with Jurkat $\mathrm{T}$ cells for $48 \mathrm{~h}$ (28).

\section{Expression of TGF- $\beta$ by TEXs}

Transforming growth factor- $\beta 1$ in exosomes has been proposed to mediate some immunosuppressive effects of TEXs. The presence of TEXs in the monocyte differentiation environment leads to the production of HLA-DR ${ }^{-/ \text {low }} \mathrm{CD}_{1}{ }^{+}$cells. TGF$\beta$ secretion by $\mathrm{CD}^{+} 4^{+} \mathrm{HLA}^{-\mathrm{DR}^{-}}$subsets was significantly increased in peripheral blood mononuclear cells isolated from melanoma patients compared to that from healthy subjects (29). It was shown that TEXs might modulate fibroblast phenotype and function via exosomal TGF- $\beta$. The mechanism associated with TGF- $\beta$ exosomal delivery is not limited to SMAD-related pathways, as it was also shown that betaglycans can accompany TGF- $\beta 1$ on the exosome surfaces, which could facilitate the effects of TGF- $\beta$ (15). HER2 targeted drug resistance is correlated with TGF- $\beta 1$ and PDL-1 levels in tumor-derived exosomes. Furthermore, exosomes that carry these molecules could result in adaptation by other drug sensitive cells (30).

\section{Expression of NKG2D Ligand/BAG6 by TEXs}

It was shown that exosomes from murine mammary cell lines can inhibit NK cell cytotoxicity (31). NKG2D is normally expressed on the surface of NK, NKT, activated $\mathrm{CD} 8^{+}$, and $\gamma \delta \mathrm{T}$ cells, and its down-regulation in tumor cells is a key mechanism through which cells evade immune surveillance. NKG2D ligands (induced self-proteins) are normally absent on the surface of cells, whereas they are up-regulated in cancers. NKG2D ligands and soluble growth factors including tumor cell secreted TGF- $\beta 1$ might affect the expression of NKG2D. In NK cells, NKG2D itself is able to trigger cytotoxicity, whereas the function of NKG2D in $\mathrm{CD}^{+} \mathrm{T}$ cells is to send co-stimulatory activating signals. In exosome studies, it was found that NKG2D ligands or TGF- $\beta 1$ on the surface of exosomes can down-regulate NKG2D receptors on effector cells. NKG2D ligands and TGF- $\beta 1$ expression might affect $\mathrm{CD} 8^{+} \mathrm{T}$ cells and NK cell activity by reducing NKG2D expression on these cells. It is obvious that TEXs harboring a ligand for NKG2D that interacts with lymphocytes do not activate $\mathrm{CD}^{+} \mathrm{T}$ cells or NK cells (32).

Blocking ULBP1-5 and MIC A/B in TEXs increases the expression of NKG2D indicating that NKG2D ligand expression in TEXs inhibits NKG2D expression on the surface of NK cells and $\mathrm{CD} 8^{+} \mathrm{T}$ cells, which leads to the suppression of their in vitro and in vivo loss of function $(33,34)$. Although, NKG2D ligands on the surface of TEXs were shown to block the activating role NKG2D, one of the NKP30-ligands named BAG6 was expressed on the surface of TEXs and as a soluble molecule; it was sown that the soluble form could promote tumor cell resistance to NKmediated cytotoxicity, whereas the exosomal form triggered NK cell activation (35).

Although most of experiments have explained the immunosuppressive effects of TEXs on diverse immune cells, they revealed that these structures can provide tumor antigens and heat shock proteins such as HSP70 on their surface which could induce protective anti-tumor immune responses. Gastper et al. 36 suggested that natural killer (NK) cells was stimulated selectively by Hsp70/Bag-4 surface-positive exosomes.

\section{Induction of Treg Population by TEXs}

Tumor derived exosomes can serve as the vehicle responsible for inducing changes in mRNA expression levels in T cells through their miRNA content (37). Human T cells co-incubated with TEXs or exosomes isolated from the plasma of patients with cancer were shown to down-regulate $\mathrm{CD} 3 \zeta$ and JAK3 expression in primary activated $\mathrm{T}$ cells and mediate the Fas/FasL-mediated apoptosis of activated $\mathrm{CD}^{+} \mathrm{T}$ cells. TEXs also promote the proliferation of $\mathrm{CD} 4+\mathrm{T}$ conventional and their conversion to $\mathrm{CD} 4{ }^{+} \mathrm{CD} 25^{\text {high }} \mathrm{FOXP} 3^{+} \mathrm{CD} 39^{+}$Tregs, which co-express IL10 and TGF- $\beta$, CTLA- 4 , and granzyme B/perforin $(27,37)$ and regulate $\mathrm{ADO}$ production by delivering $\mathrm{CD} 73$ to the Tregs (38). Thus, TEXs effectively mediate immune suppression. TEXs also increase TGF- $\beta 1$-associated phospho-SMAD2/3 and phospho-STAT3 levels and IL-10 expression in Tregs (39). T cell response to TEXs is related to surface signaling rather than internalization. Signaling might trigger $\mathrm{Ca}^{2+}$ influx or adenosine/A2A R reactions. Recent studies suggest that Tregs are potently induced by these pathways, in contrast to that observed for $\mathrm{CD}^{+}$or $\mathrm{CD}^{+}$conventional $\mathrm{T}$ cells. This confirms that TEXs could regulate efficient crosstalk between tumor cells and Tregs, which might regulate the tumor environment and immune responses (40). In Tregs, TEXs-mediated down-regulation of 
genes related to the adenosine pathway results in high expression of CD39 and CD73, as well as increased adenosine production. TEXs also induce the up-regulation of inhibitory genes in $\mathrm{CD} 4^{+}$ $\mathrm{T}$ conv cells, which results in the loss of surface CD69 and a functional decline. Tumor exosomes are not internalized by $\mathrm{T}$ cells, but signaling molecules that they carry and deliver to cell surface receptors modulate gene expression and functions in human $\mathrm{T}$ lymphocytes. Moreover, TEXs not only induce differentiation and increase expansion of Tregs but also enhance their resistance to apoptosis (39).

\section{Induction of Myeloid-Derived Suppressor Cell (MDSC) by TEXs}

Myeloid-derived suppressor cells have been identified in both human and mouse peripheral blood as a population of immature cells with the ability to suppress T-cell activation. Their accumulation in tumor-bearing mice and human cancer patients was shown to contribute to the development of cancer. Chalmin et al. (41) isolated exosomes from a mouse tumor cell line and demonstrated that the interaction between heat shock protein 72 (HSP72) on the surface of exosomes and the suppressive activity of MDSCs was mediated by the activation of STAT3. In addition, soluble factors derived from tumors increase MDSC induction through Erk pathway activation. HSP72 on the TEXs surface activates STAT3 in MDSCs through TLR $2 /$ myd 88 and the autocrine production of IL-6. At the molecular level, HSP72 in TEXs, which can act as a ligand of TLR2 in MDSCs, is responsible for the activation of MDSCs and their immune repressive ability. The production of TEXs was decreased using dimethyl amiloride, a drug used to treat high blood pressure, and in vivo efficiency of the anti-tumor drug cyclophosphamide was increased in three different mouse tumor models. Overall, the findings in both human and mouse cell lines indicate that HSP72 expression on the surface of TEXs might prevent tumor recognition by the immune system (41).

\section{Inhibition of DC Differentiation by TEXs}

Tumor derived exosomes can target $\mathrm{CD} 11 \mathrm{~b}^{+}$myeloid precursors in vivo, in mice. Moreover, TEXs prevent the differentiation of mouse myeloid progenitor cells into dendritic cells (DCs) in vitro. The addition of TEXs on the 1st day of in vitro culture was shown to significantly inhibit the differentiation of monocytes into DCs (42). Similarly, human TEXs inhibit monocyte differentiation in vitro. IL-6 and phosphorylated STAT3 levels were increased $12 \mathrm{~h}$ after stimulating myeloid precursors cells with TEXs. Moreover, TEXs were less effective in blocking the DC differentiation of monocytes isolated from the bone marrow (BM) of IL- 6 knockout mice. The addition of rIL-6 to IL-6 knockout BM cell cultures alleviated this inhibition, restoring DC differentiation. These data indicate that IL-6 from the TEXs plays a major role in blocking the differentiation of BMDCs. Mouse myeloid progenitor cells can take up exosomes and undifferentiated myeloid progenitor cells accumulate in the mouse spleen; subsequently, the differentiation of DCs was found to be inhibited. Although a small number of myeloid progenitor cells can be generated in vitro after treatment with myeloid DC exosomes, these cells do not mature and lose their ability to stimulate T-cell activation. Taken together, these results indicate that TEXs-mediated inhibition of DC differentiation might be one of the major mechanisms through which tumor cells evade the immune response, representing a major obstacle for the successful application of immunotherapy to treat cancer (43).

\section{Other Immunosuppressive Mechanisms of TEXs}

Contact between stromal and cancer cells can affect the response to treatment. Boelens et al. (44) showed that the stromal and breast cancer cells utilize paracrine and juxtacrine signaling to acquire resistance to chemotherapy and radiotherapy. The transfer of exosomes from stromal cells to breast cancer cells during their interaction was also demonstrated. RNA species in exosomes, which are largely non-coding elements, stimulate RIGI receptors and activate STAT1 signaling. In parallel, stromal cells activate NOTCH3 in breast cancer cells. Therefore, stromal cells use exosomes to stimulate and orchestrate signaling in breast cancer cell subpopulations to acquire resistance to treatment and mediate tumor recurrence (44).

Although these findings explain the immunosuppressive effects of TEXs on diverse immune cells, they also suggest that these structures can provide tumor antigens for antigen processing cells and enhance the chances of tumor antigen recognition by immune cells. Dendritic cells pulsed with TEXs can prime cytotoxic $\mathrm{T}$ cells to induce protective anti-tumor immune responses. Furthermore, high levels of Hsp70 in TEXs were reported to elicit a direct Th1-polarized immune response. In addition, NK and NKT activation by TEXs was also reported $(36,45)$.

\section{STRATEGIES FOR INCREASING IMMUNOSTIMULATORY EFFECTS OF TEXs}

According to the aforementioned results, it seems that appropriate responses from the host against TEXs will only be induced by engineering them; accordingly, several studies have been conducted and their results are proof of this claim. Here, we discuss strategies devised over the past few years to circumvent this problem, immunosuppressive properties of TEXs, along with their possible applications and limitations.

\section{Genetic Engineering}

Tumor derived exosomes could be manipulated by tumorassociated genes or some known immune boosters such as CpG DNA and TLR ligands and overexpress them to induce cellular and innate immunity; they could also be targeted using genetic materials such as siRNA, which can be introduced by electroporation into purified exosomes. Using this approach, nerve cells were previously targeted and gene silencing was confirmed (46). As a novel vaccine for cancer therapy, exosomes 
secreted by B16 melanoma cells modified to express both tumor-associated antigens and the pathogenic antigen, which increased cellular immunity against Mycobacterium tuberculosis and resulted in suppression of tumor growth in tumorbearing mice (47). Moreover, streptavidin and lactadherin expressing exosomes (SAV-exos) used in combination with biotinylated $\mathrm{CpG}$ DNA and immunization with CpG-SAVexos resulted in strong anti-tumor effects in tumor-bearing mice (48).

\section{miRNA Modification}

Exosomes secreted from cell lines that were treated with DHA (docosahexaenoic acid) were shown to express higher levels of let-7a, miR-23b, miR-27a/b, miR-21, let-7, and miR-320b, which are tumor suppressor miRNAs. Further untreated epithelial cells incubated with the exosomes are also shown to have increased levels of those miRNAs. The data showed that DHA, as an antiangiogenic factor, can affect miRNA levels in tumor exosomes. Therefore, we could target exosome signaling between tumor cells in the cancer microenvironment (49). Also, we found that Let-7i and miR-142 upregulation in TEX have notably enhancing effect on either DC maturation or CTL induction and cytokine release (50).

Moreover, ultra-filtered exosome lysates (UELs), which can be prepared for the depletion of miRNAs from exosomes, were assayed and the resulting protein extraction was used for dendritic cell activation. The results indicate that miRNAdepleted TEXs proteins might be promising agonists for the specific activation of DCs (51).

\section{Conjugation}

The binding of some molecules triggers immune cells to naturally become potent effectors. Furthermore, the binding of such activator ligands or co-stimulators to TEXs might increase their effectiveness in difference processes such as attaching to TLR ligands like CpG-DNA, Poly:IC and IL15R $\alpha$ or activation of NK cells by using NKG2D ligands. Since, exosomes produced by immature monocyte derived DC naturally present Bat 3 and nkp30 ligands on their surface, leading to diminished NK cell responses (52).

In addition, we should mention another opportunity; VAR2CSA is a malarial protein that can bind the VAR2CSAligand or chondroitin sulfate (CS) expressed in the placenta. CS is also present on a high proportion of malignant cells and could be targeted by recombinant VAR2CSA (rVAR2). Salanti showed that this rVAR2 could bind placental-type CS as well as tumor cells (PC-3, MDA-MB-231, and MyLa2059). They proposed that targeting the common CS chain, which is present on different cancer cells, might offer a novel cancer treatment strategy (53, 54) furthermore, VAR2CSA upregulated TEXs could be the next level of this vaccination procedure.

The use of exosomes for protein loading via optically reversible protein-protein interactions (EXPLORs) is a new strategy that was described for the intracellular delivery of target proteins. This occurs through the integration of a protein-protein interaction module that is controlled by blue light during endogenous exosome biogenesis (55). It has also been shown that the IFN gamma and its target gene, IRF-1 has important role in tumor cell apoptosis. TEXs released from IRF1 primed cells containing increased level of IL-15R and MHC-I and could promote antitumor immunity by CD $4+$ and CD $8+\mathrm{T}$ cells infiltration (56).

\section{HSP70/90 Overexpression (Heat Treatment)}

It has been shown that exosomes isolated from tumor cells express heat shock proteins including the cognate $71-\mathrm{kDa}$ HSP, the 70-kDa HSP4, and HSP90 alpha and beta on their surfaces (57). Moreover, tumor-derived chaperon rich cell lysates containing Hsp70, Hsp90, calreticulin, and glucose-regulated protein 94 have been reported to activate DCs (58). Bu et al. (59) demonstrated that exosomes from DCs loaded with chaperone-rich cell lysates could elicit a potent $\mathrm{T}$ cell immune response against intracranial glioma in mice. These findings suggested that chaperons are potent candidates to increase the immunostimulatory effect of TEXs.

\section{Lipid Modification and Glycosylation}

Hybrid exosomes, which are developed by fusing their membranes with liposomes (60-62), can be combined with genetic modification techniques. The interactions between the engineered exosomes and cells could be modified by changing the lipid composition or the properties of the exogenous lipids, which was shown to facilitate drug delivery or antigen presentation to immune cells located in the lymph node (62) and delivered CRISPR/Cas9 system in Mesenchymal Stem cells (MSCs) to alleviate in vivo gene editing (63). Glycosylation was identified as a promising strategy for stabilizing peptides on the surface of exosomes; this was shown to protect them from proteolytic degradation and effectively direct exosomes to specific destinations in vivo. This strategy could enhance the display of targeting peptides and generally enable and improve exosome-based therapeutics (64).

\section{Future Vesicle-Based Cancer Therapies}

As drug delivery vehicles, exosomes could be used for carrying various hemotherapeutic agents. Curcumin (natural polyphenol compound) which its anti-inflammatory effect have been shown in several studies (65-67) was packed in exosomes to enhance curcumin effectiveness $(68,69)$. Paclitaxel, doxorubicin, and temozolomide could be packed into exosomes. siRNAs or antimiRNA oligonucleotides might also be used as therapeutic cargos carried by exosomes (70). Moreover, novel technologies like nanoparticles (cationic liposomes) containing tumor RNAs, known as RNA lipoplexes (RNA-LPX), have been established. These RNA-LPXs were shown to induce immune responses (7174). An in vivo study using intravenous vaccination demonstrated the homing of RNA-LPXs to lymphoid tissues and stimulation of macrophages and DCs, which promoted $\mathrm{T}$ cell responses via IFN- $\alpha$ release and Th1 and $\mathrm{CD}^{+}$polarization (72). MSCs are particularly interesting as they localize and migrate toward 
damaged and inflammatory microenvironments including solid tumors, however they display multi-functional activities with both pro- and anti-tumor effects within the microenvironment; thus, MSC-derived exosomes could represent a potential antitumor vaccine $(75)$ or drug delivery strategy $(76,77)$.

\section{CONCLUSION}

As discussed, TEXs play a major role in tumor immune evasion and growth. In addition, they protect tumor cells from chemotherapy and immunotherapy via the efflux the chemicals and the masking of monoclonal antibody binding sites. TEXs, comprising a critical part of the tumor microenvironment, have been proposed as markers for the early diagnosis of cancers. Although evidences suggest that we should target TEXs for cancer treatment, it might be time to address this from a different point view. Based on data that we described herein, we suggest that tumor derived extracellular vesicles could be manipulated and provide cancer treatment. In this way, tumor-associated antigens, unrecognized antigens in the cytosol of TEXs, and other

\section{REFERENCES}

1. Ramachandran S, Palanisamy V. Horizontal transfer of RNAs: exosomes as mediators of intercellular communication. Wiley Interdiscip Rev RNA. (2012) 3:286-93. doi: 10.1002/wrna.115

2. Record M, Carayon K, Poirot M, Silvente-Poirot S. Exosomes as new vesicular lipid transporters involved in cell-cell communication and various pathophysiologies. Biochim Biophys Acta. (2014) 1841:108-20. doi: 10.1016/j. bbalip.2013.10.004

3. Rashed MH, Bayraktar E, GK Helal, Abd-Ellah MF, Amero P, Chavez-Reyes A, et al. Exosomes: from garbage bins to promising therapeutic targets. Int $\mathrm{J} \mathrm{Mol}$ Sci. (2017) 18:538-63. doi: 10.3390/ijms18030538

4. Hessvik NP, Llorente A. Current knowledge on exosome biogenesis and release. Cell Mol Life Sci. (2018) 75:193-208. doi: 10.1007/s00018-017-2595-9

5. Bebelman MP, Smit MJ, Pegtel DM, Baglio SR. Biogenesis and function of extracellular vesicles in cancer. Pharmacol Ther. (2018) 188:1-11. doi: 10.1016/j.pharmthera.2018.02.013

6. Stoorvogel W, Kleijmeer MJ, Geuze HJ, Raposo G. The biogenesis and functions of exosomes. Traffic. (2002) 3:321-30.

7. Théry C, Ostrowski M, Segura E. Membrane vesicles as conveyors of immune responses. Nat Rev Immunol. (2009) 9:581-93. doi: 10.1038/nri2567

8. Kowal J, Tkach M, Théry C. Biogenesis and secretion of exosomes. Curr Opin Cell Biol. (2014) 29:116-25. doi: 10.1016/j.ceb.2014.05.004

9. Andre F, Escudier B, Angevin E, Tursz T, Zitvogel L. Exosomes for cancer immunotherapy. Ann Oncol. (2004) 15:141-4.

10. Paggetti J, Haderk F, Seiffert M, Janji B, Distler U, Ammerlaan W, et al. Exosomes released by chronic lymphocytic leukemia cells induce the transition of stromal cells into cancer-associated fibroblasts. Blood. (2015) 126:1106-17. doi: 10.1182/blood-2014-12-618025

11. Willms E, Johansson HJ, Mäger I, Lee Y, Blomberg KEM, Sadik M, et al. Cells release subpopulations of exosomes with distinct molecular and biological properties. Sci Rep. (2016) 6:22519. doi: 10.1038/srep 22519

12. Zhang H, Xie Y, Li W, Chibbar R, Xiong S, Xiang J. CD4+ T cellreleased exosomes inhibit CD8+ cytotoxic T-lymphocyte responses and antitumor immunity. Cell Mol Immunol. (2011) 8:23-30. doi: 10.1038/cmi. 2010.59

13. Valadi H, Ekström K, Bossios A, Sjöstrand M, Lee JJ, Lötvall JO. Exosomemediated transfer of mRNAs and microRNAs is a novel mechanism of genetic exchange between cells. Nat Cell Biol. (2007) 9:654-9. molecules such as HSPs could be exploited in the future for cancer treatment.

\section{AUTHOR CONTRIBUTIONS}

All authors have contributed to organizing the main idea. ME and ZH helped, supported, and supervised. AT developed the idea and wrote the manuscript. FF, FS, and SM contributed in editing.

\section{FUNDING}

This work was supported by the National Institute for Medical Research Development (NIMAD; Grant No. 971113).

\section{ACKNOWLEDGMENTS}

We gratefully express our appreciation to Professor Riccardo Fodde for his helpful deliberation and consultation during this work.

14. Andre F, Schartz NE, Movassagh M, Flament C, Pautier P, Morice P, et al. Malignant effusions and immunogenic tumour-derived exosomes. Lancet. (2002) 360:295-305. doi: 10.1016/s0140-6736(02)09552-1

15. Webber J, Steadman R, Mason MD, Tabi Z, Clayton A. Cancer exosomes trigger fibroblast to myofibroblast differentiation. Cancer Res. (2010) 70:9621-30. doi: 10.1158/0008-5472.CAN-10-1722

16. Yang C, Robbins PD. The roles of tumor-derived exosomes in cancer pathogenesis. Clin Dev Immunol. (2011) 2011:1-11. doi: 10.1155/2011/842849

17. Colombo M, Raposo G, Théry C. Biogenesis, secretion, and intercellular interactions of exosomes and other extracellular vesicles. Annu Rev Cell Dev Biol. (2014) 30:255-89. doi: 10.1146/annurev-cellbio-101512-122326

18. Pfeffer SR. Two rabs for exosome release. Nat Cell Biol. (2010) 12:3-4. doi: $10.1038 /$ ncb0110-3

19. Raiborg C, Stenmark H. The ESCRT machinery in endosomal sorting of ubiquitylated membrane proteins. Nature. (2009) 458:445-52. doi: 10.1038/ nature 07961

20. Greening DW, Xu R, Ji H, Tauro BJ, Simpson RJ. A protocol for exosome isolation and characterization: evaluation of ultracentrifugation, densitygradient separation, and immunoaffinity capture methods. Methods Mol Biol. (2015) 1295:179-209. doi: 10.1007/978-1-4939-2550-6_15

21. Lötvall J, Hill AF, Hochberg F, Buzás EI, Di Vizio D, Gardiner C, et al. Minimal experimental requirements for definition of extracellular vesicles and their functions: a position statement from the International Society for Extracellular Vesicles. J Extracell Vesicles. (2014) 3:26913. doi: 10.3402/jev.v3.26913

22. Sokolova V, Ludwig A-K, Hornung S, Rotan O, Horn PA, Epple M, et al. Characterisation of exosomes derived from human cells by nanoparticle tracking analysis and scanning electron microscopy. Colloids Surf B Biointerfaces. (2011) 87:146-50. doi: 10.1016/j.colsurfb.2011.05.013

23. Théry C, Amigorena S, Raposo G, Clayton A. Isolation and characterization of exosomes from cell culture supernatants and biological fluids. Curr Protoc Cell Biol. (2006) 30:3.22.1-29.

24. Zhang Y, Liu Y, Liu H, Tang WH. Exosomes: biogenesis, biologic function and clinical potential. Cell Biosci. (2019) 9:19.

25. Abusamra AJ, Zhong Z, Zheng X, Li M, Ichim TE, Chin JL, et al. Tumor exosomes expressing Fas ligand mediate CD8+ T-cell apoptosis. Blood Cells Mol Dis. (2005) 35:169-73.

26. Kim JW, Wieckowski E, Taylor DD, Reichert TE, Watkins S, Whiteside TL. Fas ligand-positive membranous vesicles isolated from sera of patients with oral cancer induce apoptosis of activated T lymphocytes. Clin Cancer Res. (2005) 11:1010-20. 
27. Wieckowski EU, Visus C, Szajnik M, Szczepanski MJ, Storkus WJ, Whiteside TL. Tumor-derived microvesicles promote regulatory $\mathrm{T}$ cell expansion and induce apoptosis in tumor-reactive activated CD8+ T lymphocytes. J Immunol. (2009) 183:3720-30. doi: 10.4049/jimmunol.0900970

28. Taylor DD, Gerçel-Taylor C. Tumour-derived exosomes and their role in cancer-associated T-cell signalling defects. Br J Cancer. (2005) 92:305-11.

29. Valenti R, Huber V, Filipazzi P, Pilla L, Sovena G, Villa A, et al. Human tumor-released microvesicles promote the differentiation of myeloid cells with transforming growth factor-b mediated suppressive activity on T lymphocytes. Cancer Res. (2006) 66:9290-8.

30. Martinez VG, O’Neill S, Salimu J, Breslin S, Clayton A, Crown J, et al. Resistance to HER2-targeted anti-cancer drugs is associated with immune evasion in cancer cells and their derived extracellular vesicles. OncoImmunology. (2017) 6:e1362530. doi: 10.1080/2162402X.2017.1362530

31. Liu C, Yu S, Zinn K, Wang J, Zhang L, Jia Y, et al. Murine mammary carcinoma exosomes promote tumor growth by suppression of NK cell function. $J$ Immunol. (2006) 176:1375-85.

32. Clayton A, Mitchell JP, Linnane S, Mason MD, Tabi Z. Human tumorderived exosomes down-modulate NKG2D expression. J Immunol. (2008) 180:7249-58.

33. Lundholm M, Schröder M, Nagaeva O, Baranov V, Widmark A, MinchevaNilsson L, et al. Prostate tumor-derived exosomes down-regulate NKG2D expression on natural killer cells and CD8+ T Cells: mechanism of immune evasion. PLoS One. (2014) 9:e108925. doi: 10.1371/journal.pone.0108925

34. Mincheva-Nilsson L, Baranov V. Cancer exosomes and NKG2D receptorligand interactions: Impairing NKG2D-mediated cytotoxicity and anti-tumour immune surveillance. Semin Cancer Biol. (2014) 28:24-30. doi: 10.1016/j.semcancer.2014.02.010

35. Reiners KS, Topolar D, Henke A, Simhadri VR, Kessler J, Sauer M, et al. Soluble ligands for NK cell receptors promote evasion of chronic lymphocytic leukemia cells from NK cell anti-tumor activity. Blood. (2013) 121:3658-65. doi: 10.1182/blood-2013-01-476606

36. Gastpar R, Gehrmann M, Bausero MA, Asea A, Gross C, Schroeder JA, et al. Heat shock protein 70 surface-positive tumor exosomes stimulate migratory and cytolytic activity of natural killer cells. Cancer Res. (2005) 65:5238-47.

37. Muller L, Mitsuhashi M, Simms P, Gooding WE, Whiteside TL. Tumorderived exosomes regulate expression of immune function-related genes in human T cell subsets. Sci Rep. (2016) 6:20254. doi: 10.1038/srep20254

38. Schuler P, Saze Z, Hong CS, Muller L, Gillespie D, Cheng D, et al. Human $\mathrm{CD} 4+\mathrm{CD} 39+$ regulatory $\mathrm{T}$ cells produce adenosine upon co-expression of surface CD73 or contact with CD73+ exosomes or CD73+ cells. Clini Exp Immunol. (2014) 177:531-43.

39. Szajnik M, Czystowska M, Szczepanski MJ, Mandapathil M, Whiteside TL. Tumor-derived microvesicles induce, expand and up-regulate biological activities of human regulatory T cells (Treg). PLoS One. (2010) 5:e11469. doi: 10.1371/journal.pone.0011469

40. Muller L, Simms P, Hong C-S, Nishimura MI, Jackson EK, Watkins SC, et al. Human tumor-derived exosomes (TEX) regulate Treg functions via cell surface signaling rather than uptake mechanisms. OncoImmunology. (2017) 6:e1261243. doi: 10.1080/2162402X.2016.1261243

41. Chalmin F, Ladoire S, Mignot Gg, Vincent J, Bruchard Ml, Remy-Martin $\mathrm{J}-\mathrm{P}$, et al. Membrane-associated Hsp72 from tumor-derived exosomes mediates STAT3-dependent immunosuppressive function of mouse and human myeloid-derived suppressor cells. J Clin Invest. (2010) 120:457-71. doi: 10.1172/JCI40483

42. Salimu J, Webber J, Gurney M, Al-Taei S, Clayton A, Tabi Z. Dominant immunosuppression of dendritic cell function by prostate-cancer-derived exosomes. J Extracell Vesicles. (2017) 6:1368823. doi: 10.1080/20013078.2017. 1368823

43. Yu S, Liu C, Su K, Wang J, Liu Y, Zhang L, et al. Tumor exosomes inhibit differentiation of bone marrow dendritic cells. J Immunol. (2007) 178:6867-75

44. Boelens MC, Wu TJ, Nabet BY, Xu B, Qiu Y, Yoon T, et al. Exosome transfer from stromal to breast cancer cells regulates therapy resistance pathways. Cell. (2014) 159:499-513. doi: 10.1016/j.cell.2014.09.051

45. Gu X, Erb U, Büchler MW, Zöller M. Improved vaccine efficacy of tumor exosome compared to tumor lysate loaded dendritic cells in mice. Int J Cancer. (2015) 136:74-84. doi: 10.1002/ijc.29100
46. Alvarez-Erviti L, Seow Y, Yin H, Betts C, Lakhal S, Wood MJA. Delivery of siRNA to the mouse brain by systemic injection of targeted exosomes. Nat Biotechnol. (2011) 29:341-5. doi: 10.1038/nbt.1807

47. Koyama Y, Ito T, Hasegawa A, Eriguchi M, Inaba T, Ushigusa T, et al. Exosomes derived from tumor cells genetically modified to express Mycobacterium tuberculosis antigen: a novel vaccine for cancer therapy. Biotechnol Lett. (2016) 38:1857-66.

48. Morishita M, Takahashi Y, Matsumoto A, Nishikawa M, Takakura Y. Exosome-based tumor antigens-adjuvant co-delivery utilizing genetically engineered tumor cell-derived exosomes with immunostimulatory CpG DNA. Biomaterials. (2016) 111:55-65. doi: 10.1016/j.biomaterials.2016.09.031

49. Hannafon BN, Carpenter KJ, Berry WL, Janknecht R, Dooley WC, Ding W-Q. Exosome-mediated microRNA signaling from breast cancer cells is altered by the anti-angiogenesis agent docosahexaenoic acid (DHA). Mol Cancer. (2015) 14:133. doi: 10.1186/s12943-015-0400-7

50. Taghikhani A, Hassan ZM, Ebrahimi M, Moazzeni. microRNA modified tumo derived exosomes as novel tools for maturation of dendritic cells. J Cell Physiol. (2018) 234:9417-27. doi: 10.1002/jcp.27626

51. Que R-S, Lin C, Ding G-P, Wu Z-R, Cao L-P. Increasing the immune activity of exosomes: the effect of miRNA-depleted exosome proteins on activating dendritic cell/cytokine-induced killer cells against pancreatic cancer. J Zhejiang Univ Sci B. (2016) 17:352-60. doi: 10.1631/jzus.B1500305

52. Gehrmann U, Näslund TI, Hiltbrunner S, Larssen P, Gabrielsson S. Harnessing the exosome-induced immune response for cancer immunotherapy. Semin Cancer Biol. (2014) 28:58-67. doi: 10.1016/j.semcancer.2014.05.003

53. Daugaard M. Targeting Common but Complex Proteoglycans on Breast Cancer Cells and Stem Cells Using Evolutionary Refined Malaria Proteins. Technical Report. Vancouver, BC: The University of British Columbia (2017).

54. Salanti A. Targeting Common but Complex Proteoglycans on Breast Cancer Cells and Stem Cells Using Evolutionary Refined Malaria Proteins. Technical Report. Copenhagen: University of Copenhagen (2015).

55. Yim N, Ryu S-W, Choi K, Lee KR, Lee S, Choi H, et al. Exosome engineering for efficient intracellular delivery of soluble proteins using optically reversible protein-protein interaction module. Nat Commun. (2016) 7:12277. doi: $10.1038 /$ ncomms 12277

56. Yang M-Q, Du Q, Varley PR, Goswami J, Liang Z, Wang R, et al. Interferon regulatory factor 1 priming of tumour-derived exosomes enhances the antitumour immune response. Br J Cancer. (2018) 118:62-71. doi: 10.1038/ bjc. 2017.389

57. Burke M, Choksawangkarn W, Edwards N, Ostrand-Rosenberg S, Fenselau C. Exosomes from myeloid-derived suppressor cells carry biologically active proteins. J Proteome Res. (2013) 13:836-43. doi: 10.1021/pr400879c

58. Zeng Y, Graner MW, Katsanis E. Chaperone-rich cell lysates, immune activation and tumor vaccination. Cancer Immunol Immunother. (2005) 55:329-38.

59. Bu N, Wu H, Zhang G, Zhan S, Zhang R, Sun H, et al. Exosomes from dendritic cells loaded with chaperone-rich cell lysates elicit a potent $\mathrm{T}$ cell immune response against intracranial glioma in mice. J Mol Neurosci. (2015) 56:631-43. doi: 10.1007/s12031-015-0506-9

60. Harisa GI, Badran MM, Alanazi FK, Attia SM. Crosstalk of nanosystems induced extracellular vesicles as promising tools in biomedical applications. J Membr Biol. (2017) 250:605-16. doi: 10.1007/s00232-017-0003-X

61. Luan X, Sansanaphongpricha K, Myers I, Chen H, Yuan H, Sun D. Engineering exosomes as refined biological nanoplatforms for drug delivery. Acta Pharmacol Sin. (2017) 38:754-63. doi: 10.1038/aps.2017.12

62. Sato YT, Umezaki K, Sawada S, Mukai S-A, Sasaki Y, Harada N, et al Engineering hybrid exosomes by membrane fusion with liposomes. Sci Rep. (2016) 6:21933. doi: 10.1038/srep21933

63. Lin Y, Wu J, Gu W, Huang Y, Tong Z, Huang L, et al. Exosome-liposome hybrid nanoparticles deliver CRISPR/Cas9 system in MSCs. Adv Sci. (2018) 5:1700611-9. doi: 10.1002/advs.201700611

64. Hung ME, Leonard JN. Stabilization of exosome-targeting peptides via engineered glycosylation. J Biol Chem. (2015) 290:8166-72. doi: 10.1074/jbc. M114.621383

65. Chin K-Y. The spice for joint inflammation: anti-inflammatory role of curcumin in treating osteoarthritis. Drug Des Dev Ther. (2016) 10:3029-42.

66. Fan Z, Yao J, Li Y, Hu X, Shao H, Tian X. Anti-inflammatory and antioxidant effects of curcumin on acute lung injury in a rodent model of intestinal 
ischemia reperfusion by inhibiting the pathway of NF-Kb. Int J Clin Exp Pathol. (2015) 8:3451-9.

67. Ferreira VH, Nazli A, Dizzell SE, Mueller K, Kaushic C. The antiinflammatory activity of curcumin protects the genital mucosal epithelial barrier from disruption and blocks replication of HIV-1 and HSV-2. PLoS One. (2015) 10:e0124903. doi: 10.1371/journal.pone.012 4903

68. Sun D, Zhuang X, Grizzle W, Miller D, Zhang H-G. Abstract 4446: a novel nanoparticle drug delivery system: the anti-inflammatory activity of curcumin is enhanced when encapsulated in exosomes. Cancer Res. (2011) 71(8 Suppl.):4446.

69. Trotta T, Panaro MA, Prifti E, Porro C. Modulation of biological activities in glioblastoma mediated by curcumin. Nutr Cancer. (2019) 71:1241-53. doi: $10.1080 / 01635581.2019 .1604978$

70. Yang Z, Xie J, Zhu J, Kang C, Chiang C, Wang X, et al. Functional exosome-mimic for delivery of siRNA to cancer: in vitro and in vivo evaluation. J Control Release. (2016) 243:160-71. doi: 10.1016/j.jconrel.2016. 10.008

71. Dow S. Liposome-nucleic acid immunotherapeutics. Expert Opin Drug Deliv. (2008) 5:11-24. doi: 10.1517/17425247.5.1.11

72. Kranz LM, Diken M, Haas H, Kreiter S, Loquai C, Reuter KC, et al. Systemic RNA delivery to dendritic cells exploits antiviral defence for cancer immunotherapy. Nature. (2016) 534:396-401. doi: 10.1038/nature18300

73. Mizrahy S, Hazan-Halevy I, Landesman-Milo D, Peer D. Advanced strategies in immune modulation of cancer using lipid-based nanoparticles. Front Immunol. (2017) 8:69. doi: 10.3389/fimmu.2017.00069

74. Oberli MA, Reichmuth AM, Dorkin JR, Mitchell MJ, Fenton OS, Jaklenec A, et al. Lipid nanoparticle assisted mRNA delivery for potent cancer immunotherapy. Nano Lett. (2016) 17:1326-35. doi: 10.1021/acs.nanolett. $6 \mathrm{~b} 03329$

75. Bruno S, Collino F, Deregibus MC, Grange C, Tetta C, Camussi G. Microvesicles derived from human bone marrow mesenchymal stem cells inhibit tumor growth. Stem Cells Dev. (2012) 22:758-71. doi: 10.1089/scd. 2012.0304

76. Belmar-Lopez C, Mendoza G, Oberg D, Burnet J, Simon C, Cervello I, et al. Tissue-derived mesenchymal stromal cells used as vehicles for anti-tumor therapy exert different in vivoeffects on migration capacity and tumor growth. BMC Med. (2013) 11:139. doi: 10.1186/1741-7015-11-139

77. Rani S, Ryan AE, Griffin MD, Ritter T. Mesenchymal stem cell-derived extracellular vesicles: toward cell-free therapeutic applications. Mol Ther. (2015) 23:812-23.

78. Xiang X, Poliakov A, Liu C, Liu Y, Deng Z, Wang J, et al. Induction of myeloid derived suppressor cells by tumor exosomes. Int J Cancer. (2009) 124:2621-33. doi: $10.1002 / \mathrm{ijc} .24249$

Conflict of Interest: The authors declare that the research was conducted in the absence of any commercial or financial relationships that could be construed as a potential conflict of interest.

Copyright (®) 2020 Taghikhani, Farzaneh, Sharifzad, Mardpour, Ebrahimi and Hassan. This is an open-access article distributed under the terms of the Creative Commons Attribution License (CC BY). The use, distribution or reproduction in other forums is permitted, provided the original author(s) and the copyright owner(s) are credited and that the original publication in this journal is cited, in accordance with accepted academic practice. No use, distribution or reproduction is permitted which does not comply with these terms. 